\title{
On the dependence of the component counting process of a uniform random variable
}

\author{
Joseph Squillace*
}

\begin{abstract}
We are concerned with the general problem of proving the existence of joint distributions of two discrete random variables $M$ and $N$ subject to infinitely many constraints of the form $\mathbb{P}(M=i, N=j)=0$. In particular, the variable $M$ has a countably infinite range and the other variable $N$ is uniformly distributed with finite range. The constraints placed on the joint distributions will require, for most elements $j$ in the range of $N, \mathbb{P}(M=i, N=j)=0$ for infinitely many values of $i$ in the range of $M$, where the corresponding values of $i$ depend on $j$. To prove the existence of such joint distributions, we apply a theorem proved by Strassen on the existence of joint distributions with prespecified marginal distributions.

We consider some combinatorial structures that can be decomposed into components. Given $n \in \mathbb{N}$, consider an assembly, multiset, or selection $A_{n}$ among elements of $\{1,2, \ldots, n\}$, and consider a uniformly distributed random variable $N(n)$ on $A_{n}$. For each $i \leq n$, denote by $C_{i}(n)$ the number of components of $N(n)$ of size $i$ so that $\sum_{i \leq n} i C_{i}(n)=n$. In each of these combinatorial structures, there exists infinitely many processes $\left(\left(Z_{i}(n, x)\right)_{i \leq n}\right)_{x}$, indexed by a real parameter $x$, consisting of nonnegative independent variables $\left(Z_{i}(n, x)\right)_{i \leq n}$ such that the distribution of the vector $\left(C_{i}(n)\right)_{i \leq n}$ equals the distribution of the vector $\left(Z_{i}(n, x)\right)_{i \leq n}$ conditional on the event $\left\{\sum_{i \leq n} i Z_{i}(n, x)=n\right\}$. Let $M(n, x)$ denote a random variable whose components are given by $\left(Z_{i}(n, x)\right)_{i \leq n}$. We introduce the notion of pivot mass which is then combined with Strassen's work to provide couplings of $M(n, x)$ and $N(n)$ with desired properties. For each of these combinatorial structures, we prove that there exists a real number $x(n)$ for which we can couple $M(n, x)$ and $N(n)$ with $\sum_{i<n}\left(C_{i}(n)-Z_{i}(n, x)\right)^{+} \leq 1$ when $x>x(n)$. We are providing a partial answer to the question "how much dependence is there in the process $\left(C_{i}(n)\right)_{i \leq n}$ ?"
\end{abstract}

Keywords: component counting process; couplings; marriage theorem; pivot mass. MSC2020 subject classifications: $60 \mathrm{C} 05$.

Submitted to ECP on May 10, 2020, final version accepted on May 2, 2021.

Supersedes arXiv: 1910.12841.

\section{Introduction}

Our results regard the component counting process of a uniform random variable in a combinatorial structure, and these results are provided by establishing the existence of couplings of random variables.

\footnotetext{
${ }^{*}$ University of Rhode Island, United States of America. E-mail: josephps@uri.edu
} 
Definition 1.1. Let $X$ and $Y$ be random variables defined on probability spaces $\left(\Omega_{X}, \mathcal{F}_{X}\right.$, $\left.\mathbb{P}_{X}\right)$ and $\left(\Omega_{Y}, \mathcal{F}_{Y}, \mathbb{P}_{Y}\right) .{ }^{1}$ A coupling of $X$ and $Y$ is a probability space $(\Omega, \mathcal{F}, \mathbb{P})$ in which there exists random variables $X^{\prime}$ and $Y^{\prime}$ such that $X^{\prime}$ has the same distribution as $X$ and $Y^{\prime}$ has the same distribution as $Y{ }^{2}$

Given a natural number $n$, this paper is concerned with combinatorial structures $A_{n}$ on the set $[n]:=\{1,2, \ldots, n\}$ that can be decomposed into components (e.g., the components of a permutation are its cycles). The combinatorial structures considered in this paper are known as assemblies, multisets and selections, and these structures are outlined in $\S 1.1$. Given a uniform random variable $N(n) \sim \operatorname{Unif}\left(A_{n}\right)$, let $C_{i}(n)$ denote the number of components of $N(n)$ of size $i, 1 \leq i \leq n$. The sequence $\left(C_{i}(n)\right)_{i \leq n}$ is called the component counting process of $N(n)$; the variables $C_{i}(n), 1 \leq i \leq n$, are dependent since $\sum_{i \leq n} i C_{i}(n)=n$. Our goal is to measure the amount of dependence in this sequence by showing that it is dominated by a related sequence $\left(Z_{i}\right)_{i}$ of independent variables $Z_{i}$ in the following sense. ${ }^{3}$

Definition 1.2. Let $n \in \mathbb{N}$. Given random variables $M(n)$ and $N(n)$ with components $Z_{i}(n)$ and $C_{i}(n), 1 \leq i \leq n$, respectively, the variable $M(n) d$-dominates $N(n)$ if there exists a coupling of $M(n)$ and $N(n)$ such that

$$
\sum_{i \leq n}\left(C_{i}(n)-Z_{i}(n)\right)^{+} \leq d, \quad \text { always }
$$

Condition (1.1) is equivalent to the equation $\mathbb{P}\left(\sum_{i \leq n}\left(C_{i}(n)-Z_{i}(n)\right)^{+} \leq d\right)=1$. The goal of this paper is to show that a uniform variable $N$ is 1-dominated by a particular variable $M$.

There is a natural combinatorial setup [3], with an $N(n)$ based on uniformly chosen instances, and related exponential families of independent variables $Z_{i}(n, x), 1 \leq i \leq n$; the free parameter $x[3,4]$ corresponds to Cramer tilting. The combinatorial $N(n)$ and the independent variables $Z_{i}(n, x)$ are related by the conditioning relation, given by equation (1.2) below. Let $M(n, x)$ denote a random variable with $i$ parts of size $Z_{i}(n, x)$. Now as $x$ increases (to infinity for assemblies, to 1 for multisets and selections), the $Z_{i}$ converge in distribution to the supremum of their support. Thus under any coupling, the terms $\left(C_{i}(n)-Z_{i}(n, x)\right)^{+}$converge in distribution to 0 and $\mathbb{P}\left(\sum_{i \leq n}\left(C_{i}(n)-Z_{i}(n, x)\right)^{+} \leq 1\right)$ tends to 1 , where $(\cdot)^{+}$denotes the positive part. The main result of this paper, Theorem 1.4, strengthens this fact since it provides the existence of a coupling of $N(n)$ and $M(n, x)$ such that $M(n, x)$ 1-dominates $N(n)$ for sufficiently large values of $x$. Moreover, it will be shown that $M(n, x)$ does not 0-dominate $N(n)$ (i.e., we do not have $Z_{i}(n, x) \leq C_{i}(n)$ for all $\left.i \leq n\right)$.

The notion of $d$-domination is motivated by a conjecture proposed by Richard Arratia in $\S 2.2$ of [1], which we now describe. Consider a uniformly distributed variable $N(n) \in$ $[n]$ with prime factorization $N(n)=\prod_{p \leq n} p^{C_{p}(n)}$. It can be shown that the prime power process $\left(C_{p}(n)\right)_{p \leq n}$ converges in distribution to a process $\left(Z_{p}\right)_{p \leq n}$ of independent variables where $Z_{p}$ is a geometric random variable of parameter $\frac{1}{p}$ and range $\mathbb{Z}_{\geq 0}$, for each prime $p \leq n$. Defining $M(n)=\prod_{p \leq n} p^{Z_{p}}$, we state Arratia's conjecture.

Conjecture 1.3 (Arratia). For all $n \geq 1$, it is possible to construct $N(n)$ uniformly

\footnotetext{
${ }^{1}$ The probability measure $\mathbb{P}_{X}$ is defined by $\mathbb{P}_{X}(i)=\mathbb{P}(X=i)$.

${ }^{2}$ For each of the random variables $X$ considered in this paper, $X$ and $X^{\prime}$ will share the same range. Thus, when describing a particular coupling of $X$ and $Y$, we often write $X$ and $Y$ instead of $X^{\prime}$ and $Y^{\prime}$, respectively.

${ }^{3}$ Note that only component counting processes $\left(C_{i}(n)\right)_{i \leq n}$ satisfy the condition $\sum_{i \leq n} i C_{i}(n)=n$ for all $n \in \mathbb{N}$. In general, variables denoted by $Z_{i}(n), i \in \mathbb{N}$, will be independent, so we cannot assume $\sum_{i \leq n} i Z_{i}(n)=n$ for any $n \in \mathbb{N}$.
} 
The component counting process of a uniform random variable

distributed from 1 to $n, M(n)$ and a prime $P(n)$ such that

$$
\text { always } N(n) \text { divides } M(n) P(n) \text {. }
$$

Equivalently, the conjecture states that there exists a coupling of $M(n)$ and $N(n)$ such that we always have $\sum_{p \leq n}\left(C_{p}(n)-Z_{p}\right)^{+} \leq 1$, and this was the motivation for the definition of $d$-domination. However, Arratia's conjecture does not fall within the combinatorial settings considered in this paper due to the fact that the prime power process $\left(C_{p}(n)\right)_{p \leq n}$ does not satisfy the equation $\sum_{p \leq n} p C_{p}(n)=n$.

The combinatorial structures listed in $\S 1.1$ provide the frameworks in which we obtain our couplings. Theorem 1.4, the main result of this paper, is stated in $\S 1.2$. In $\S 2$, we describe how our constraints force a significant proportion of the entries of a prospective joint mass distribution of our variables to be 0 . In $\S 3$, we introduce the notion of pivot mass, which depends on the constraints placed on the desired joint distribution. Some properties of the pivot mass are proved in $\S 3$ and $\S 4$. In $\S 5$, we apply results on the pivot mass and a theorem proved by Strassen to prove Theorem 1.4, thereby proving the existence of our couplings.

\subsection{Three major combinatorial structures}

All couplings constructed in this paper involve a uniform random variable in any one of the following three combinatorial classes. An assembly $A_{n}$ is an example of a combinatorial structure in which the set $[n]$ is partitioned into blocks and for each block of size $i$ one of $m_{i}$ possible structures is chosen. A multiset $A_{n}$ is a pair $([n], m)$, where $m: A \rightarrow \mathbb{N}$ is a function that gives the multiplicity $m(a)$ of each element $a \in[n]$. Equivalently (see Meta-example 2.2 of $\S 2.2$ of [2]), the integer $n$ is partitioned into parts, and for each part of size $i$, one of the $m_{i}$ objects of weight $i$ is chosen. Selections are similar to multisets, but now we require all parts to be distinct. To simplify the notation, let us define $k_{n}:=\# A_{n}$ for each of these structures.

\subsection{Couplings of random variables}

In each of the assembly, multiset, and selection settings, our methods of arriving at our desired couplings are similar. We start by considering $N(n) \sim \operatorname{Unif}\left(A_{n}\right)$. Given $i \leq n$, if we denote by $C_{i}(n)$ the number components of $N(n)$ of size $i$, then $0 \leq C_{i}(n) \leq n$ and $\sum_{i \leq n} i C_{i}(n)=n$. In these combinatorial settings, there exists an infinite family $\left(\left(Z_{i}(n, x)\right)_{i \leq n}\right)_{x}$, parametrized by positive values of $x$ (specifically, $x>0$ for assemblies, $x \in(0,1)$ for multisets, and $x \in(0, \infty)$ for selections) of infinite sequences $\left(Z_{i}(n, x)\right)_{i \leq n}$ of nonnegative integer-valued independent random variables $Z_{i}(n, x)$ for which

$$
\mathcal{L}\left(C_{1}(n), \ldots, C_{n}(n)\right)=\mathcal{L}\left(Z_{1}(n, x), \ldots, Z_{n}(n, x) \mid \sum_{i \leq n} i Z_{i}(n, x)=n\right)
$$

( $\$ 2.3$ of [2]). Equation (1.2) states that the joint distribution of the vector $\left(C_{i}(n)\right)_{i \leq n}$ is equal to the joint distribution of the vector $\left(Z_{i}(n, x)\right)_{i \leq n}$ conditional on the event $\left\{\sum_{i \leq n} i Z_{i}(n, x)=n\right\}$. For a fixed $x$, we consider another random variable $M(n, x)$ whose component counts ${ }^{4}$ are given by $\left(Z_{i}(n, x)\right)_{i \leq n}$, so the distribution of $M(n, x)$ is determined by the independent process $\left(Z_{i}(n, x)\right)_{i \leq n}$.

The main result of this paper is the following theorem which deals with the case $d=1$; this theorem asserts the existence of a finite $x(n)$ such that, for all $x>x(n)$,

\footnotetext{
${ }^{4}$ For fixed $x$, since the variables $Z_{i}(n, x), i \leq n$, are independent, it is not always true that $\sum_{i \leq n} i Z_{i}(n, x)=$ $n$. Therefore, the variable $M(n, x)$ does not always correspond to an element of $A_{n}$.
} 
couplings exist in which $M(n, x)$ 1-dominates $N(n) .^{5}$

Theorem 1.4. Let $n \in \mathbb{N}$ and suppose $A_{n}$ denotes an assembly, multiset, or a selection among elements of $[n]$. Given $N(n) \sim \operatorname{Unif}\left(A_{n}\right)$ with component counting process $\left(C_{i}(n)\right)_{i \leq n}$, there exists a positive real number $x(n)$ for which, when $x>x(n)$, there exists a process $\left(Z_{i}(n, x)\right)_{i \leq n}$ of non-negative independent random variables satisfying (1.2) such that $M(n, x)$ 1-dominates $N(n)$.

It is worth noting that $M(n, x)$ does not 0 -dominate $N(n)$ due to the fact that the quantity $\mathbb{P}\left(Z_{i}(n, x)=0\right.$ for all $\left.i \leq n\right)$ is positive for each of the combinatorial structures considered in this paper (see $\S 4$ and apply independence of the $Z_{i}$ 's); however, the $C_{i}(n)^{\prime}$ 's are never all 0 due to the constraint $\sum_{i \leq n} i C_{i}(n)=n$.

\section{The joint mass distribution of $(M(n, x), N(n))$}

For some fixed value of $x$, if we are to successively construct a joint probability mass function $p(\cdot, \cdot)$ such that $M(n, x)$ 1-dominates $N(n)$, we must ensure that $\mathbb{P}(M(n, x)=\cdot, N(n)=\cdot)=0$ when $\sum_{i \leq n}\left(C_{i}(n)-Z_{i}(n, x)\right)^{+}>1$. We can index the joint distribution by using the range of $N(n)$ and the range of $M(n, x)$ for the column labels and row labels, respectively. In particular, we can label the columns with the range of $\left(C_{i}(n)\right)_{i \leq n}$ in lexicographic order. Since we have infinitely many row labels, for each $m \in \mathbb{Z}_{\geq 0}$, we apply the lexicographic ordering on all elements $\left(m_{1}, \ldots, m_{n}\right) \in\left(\mathbb{Z}_{\geq 0}\right)^{n}$ with $\sum_{i \leq n} m_{i}=m$, starting with $m=0$ (we start with $m=0$ since the $Z_{i}(n, x)^{\prime}$ 's are non-negative). With respect to this ordering, we will often enumerate the columns by $1,2, \ldots, k_{n}$ and the rows by $1,2, \ldots$.

In each of these three settings, there are additional constraints on any joint probability mass function of $M(n, x)$ and $N(n)$ since the marginal distributions are known. In particular, the sum along column $N(n)=j, 1 \leq j \leq k_{n}$, is $\mathbb{P}(N(n)=j)=1 / k_{n}$; and the sum along the row $M(n, x)=m, m \in \mathbb{N}$, labeled $\left(Z_{i}(n, x)\right)_{i \leq n}=\left(m_{i}\right)_{i \leq n}$ is $\mathbb{P}\left(\left(Z_{i}(n, x)\right)_{i \leq n}=\left(m_{i}\right)_{i \leq n}\right)=\prod_{i \leq n} \mathbb{P}\left(Z_{i}(n, x)=m_{i}\right)$, where the latest equation is due to the independence of the process $\left(Z_{i}(n, x)\right)_{i \leq n}$.

\section{Pivot mass}

Given columns $j$ and $k$, with corresponding components $\left(C_{i}(n)\right)_{i \leq n}$ and $\left(C_{i}^{\prime}(n)\right)_{i \leq n}$, we seek a way to compare the corresponding sets of row labels in which column $j$ or $k$ must be 0 . Any of our desired couplings has the property that column $\left(C_{i}(n)\right)_{i \leq n}$ has a zero in row $\left(Z_{i}(n, x)\right)_{i \leq n}$ when the constraint $\sum_{i \leq n}\left(C_{i}\left((n)-Z_{i}(n, x)\right)^{+} \leq 1\right.$ is violated, so we compare the probability measures of the sets $\left\{\left(Z_{i}(n, x)\right)_{i \leq n}: \sum_{i \leq n}\left(C_{i}(n)-Z_{i}(n, x)\right)^{+}>1\right\}$ and $\left\{\left(Z_{i}(n, x)\right)_{i \leq n}: \sum_{i \leq n}\left(C_{i}^{\prime}(n)-\right.\right.$ $\left.\left.Z_{i}(n, x)\right)^{+}>1\right\}$. I.e., since we seek couplings of $M(n, x)$ and $N(n)$ for which $M(n, x)$ 1-dominates $N(n)$, we measure the probability that $M(n, x)$ takes on a value $i$ for which column $j$ or $k$ has a required 0 in row $i$. This motivates the following definition.

Definition 3.1. We call the pair $(i, j)$, corresponding to the $i$ th row label $\left(Z_{i}(x)\right)_{i \leq n}$ and the $j$ th column label $\left(C_{i}(n)\right)_{i \leq n}$, a pivot if $\sum_{i \leq n}\left(C_{i}(n)-Z_{i}(x)\right)^{+}>1$. Denote the set of all pivots by $P$. The pivot mass in column $N(n)=j$ is defined as

$$
\mathcal{P M}(j)=\mathcal{P M}_{(n, x)}(j):=\sum_{i:(i, j) \in P} \mathbb{P}(M(n, x)=i) .
$$

\footnotetext{
${ }^{5}$ To simplify the notation, we will sometimes (Figure 2, Theorem 3.3, and $\left.\S \S 4-5\right)$ replace $Z_{i}(n, x)$ with $Z_{i}$, replace $C_{i}(n)$ with $C_{i}$, replace $N(n)$ with $N$, and replace $M(n, x)$ with $M$.
} 
The component counting process of a uniform random variable

Given a subset $L(n)$ of column labels of $[n]$, the pivot mass in $L(n)$ is defined as

$$
\mathcal{P} \mathcal{M}(L(n))=\mathcal{P} \mathcal{M}_{(n, x)}(L(n)):=\sum_{\substack{i:(i, j) \in P \\ \forall j \in L(n)}} \mathbb{P}(M(n, x)=i) .
$$

Theorem 3.3 gives a formula for $\mathcal{P} \mathcal{M}(j)$. Fortunately, due to the role of the parameter $x$, it is not necessary to derive a formula for $\mathcal{P} \mathcal{M}(L(n))$ in order to prove Theorem 1.4. The fact that $\mathcal{P} \mathcal{M}(L(n)) \leq \mathcal{P} \mathcal{M}(j)$ for any $j \in L(n)$ will be sufficient. Figure 1 shows some of the features of our desired couplings.

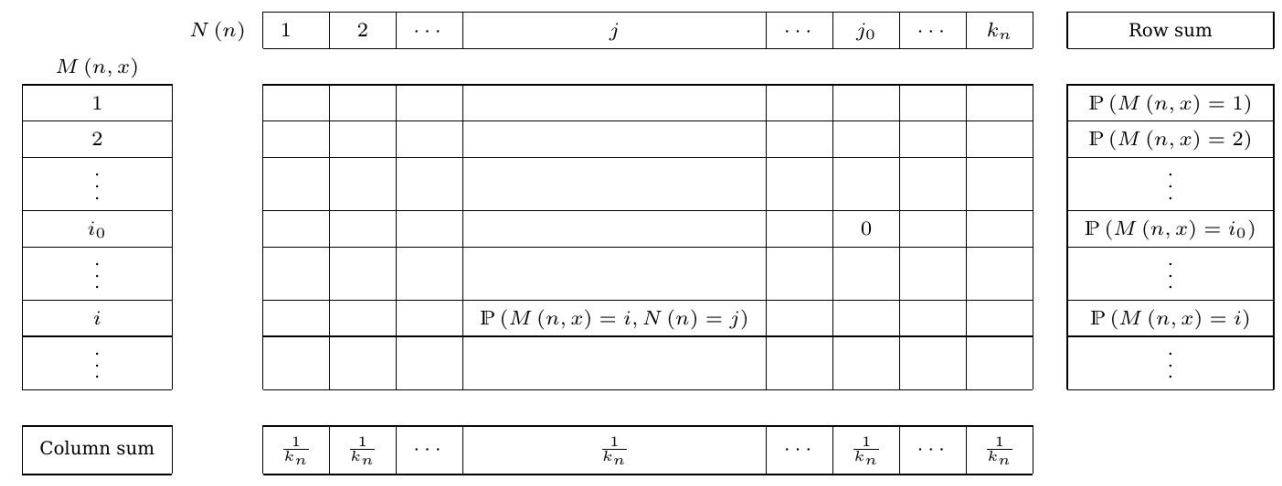

Figure 1: If $\left(i_{0}, j_{0}\right)$ is a pivot, then our desired joint distribution table should have a 0 in the $\left(i_{0}, j_{0}\right)$ entry.

Example 3.2. Fix $n=3$ and consider the assembly $A_{3}=S_{3}$ of permutations of $\{1,2,3\}$. The elements of $S_{3}$ are $1,(12),(13),(23),(123),(132)$, and their respective component counts are $(3,0,0),(1,1,0),(1,1,0),(1,1,0),(0,0,1),(0,0,1)$. Some key features of a desired joint mass distribution of $(M(3, x), N(3))$ are given in Figure 2.
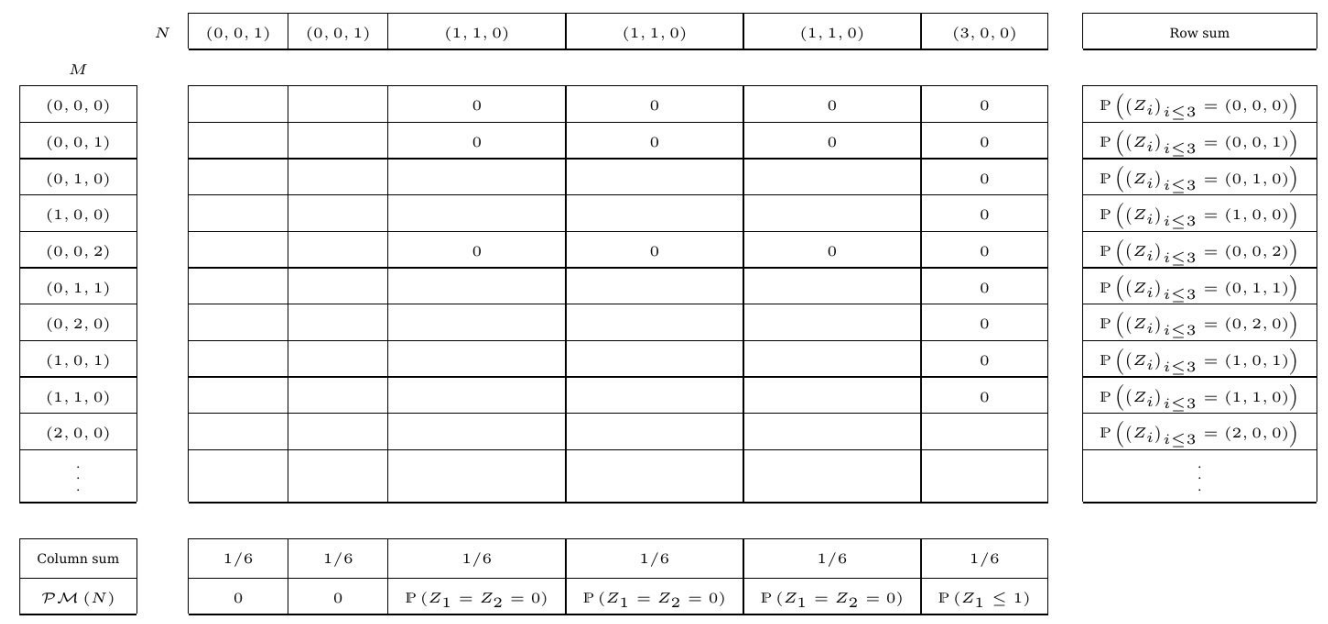

Figure 2: A desired coupling of $M(3, x)$ and $N(3)$ should have a zero at any location $\left(\left(Z_{i}(3, x)\right)_{i \leq 3},\left(C_{i}(3)\right)_{i \leq 3}\right)$ satisfying $\sum_{i \leq 3}\left(C_{i}(3)-Z_{i}(3, x)\right)^{+}>1$.

Each column with a pivot contains infinitely many pivots. E.g., in Figure 2, column $(3,0,0)$ has a pivot in any row of the form $(a, b, c)$ with $a \in\{0,1\}, b, c \geq 0$. Columns labeled $(1,1,0)$ have a pivot in any row of the form $(0,0, l)$ for any $l \in \mathbb{Z}_{\geq 0}$. Moreover, 
note that 0-domination would require each entry in the first row to be 0 ; however, each row mass is positive for all of the combinatorial structures considered in this paper.

The following theorem plays a key role in the proof of Theorem $1.4 .{ }^{6}$ For convenience, in the proof of the following theorem, we simplify the notation by writing $C_{i}(n)=C_{i}$, $Z_{i}(n, x)=Z_{i}, M(n, x)=M$ and $N(n)=N$. Moreover, the notion of pivot mass introduced in this section may be generalized; in a particular setting, one should define pivot mass based on the constraints required of their desired coupling. It is both a combinatorial and probabilistic object since it is a sum of probability masses indexed by the counting constraint $\sum_{i \leq n}\left(C_{i}(n)-Z_{i}(n, x)\right)^{+} \leq 1$.

Theorem 3.3 (Pivot Mass Formula for 1 Column). Consider a fixed column label $N(n) \in$ $A_{n}$ and denote its component counting process by $\left(C_{i}(n)\right)_{i \leq n}$. Its pivot mass is

$$
\begin{aligned}
\mathcal{P M}(N(n))= & 1-\sum_{j \leq n}\left(1_{\left\{C_{j}>0\right\}}\left(1-\mathbb{P}\left(Z_{j} \leq C_{j}-2\right)\right) \prod_{\substack{i \neq j, i \leq n}}\left(1-\mathbb{P}\left(Z_{i} \leq C_{i}-1\right)\right)\right) \\
& +\left(\sum_{i \leq n} 1_{\left\{C_{i}>0\right\}}-1\right) \prod_{i \leq n}\left(1-\mathbb{P}\left(Z_{i} \leq C_{i}-1\right)\right) .
\end{aligned}
$$

Proof. Given $1 \leq j \leq n$, let $\overrightarrow{e_{j}}$ denote the row vector of length $n$ whose $j$ th entry is 1 and whose other entries are 0 . Given two vectors $\left(a_{i}\right)_{i \leq n},\left(b_{i}\right)_{i \leq n}$ in $\mathbb{R}^{n}$, we write $\left(a_{i}\right)_{i \leq n} \leq\left(b_{i}\right)_{i \leq n}$ if $a_{i} \leq b_{i}$ for each $i \leq n$. Since $\sum_{k=1}^{\infty} \mathbb{P}(M(n, x)=k)=1$, we have

$$
\mathcal{P M}(N)=1-\sum_{k:(k, N) \notin P} \mathbb{P}(M=k) .
$$

We have the event equality

$$
\{(M, N) \notin P\}=\left\{\exists j \leq n:\left(Z_{i}\right)_{i \leq n} \geq\left(C_{i}\right)_{i \leq n}-\overrightarrow{e_{j}} \cdot 1_{\left\{C_{j}>0\right\}}\right\}
$$

since the pair $(M, N)$ is a not pivot if and only if $Z_{i} \geq C_{i}$ for all $i$ except possibly one value $j$ with $Z_{j}=C_{j}-1$. Since each $Z_{i}, 1 \leq i \leq n$, is nonnegative, we can only have $Z_{j}=C_{j}-1$ when $C_{j}>0$. Note that if $Z_{i} \geq C_{i}$ for all $i$, then any $j$ satisfies $\left(Z_{i}\right)_{i \leq n} \geq\left(C_{i}\right)_{i \leq n}-\overrightarrow{e_{j}} \cdot 1_{\left\{C_{j}>0\right\}}$. On the other hand, if there exists a value $j$ for which $Z_{j}=C_{j}-1$ and $Z_{i} \geq C_{i}$ for all $i \neq j$, then $\left(Z_{i}\right)_{i \leq n} \geq\left(C_{i}\right)_{i \leq n}-\overrightarrow{e_{j}} \cdot 1_{\left\{C_{j}>0\right\}}$. Therefore, the right hand side of equation (3.1) is

$$
1-\sum_{k:(k, N) \notin P} \mathbb{P}(M=k)=1-\mathbb{P}\left(\exists j \leq n:\left(Z_{i}\right)_{i \leq n} \geq\left(C_{i}\right)_{i \leq n}-\overrightarrow{e_{j}} \cdot 1_{\left\{C_{j}>0\right\}}\right) .
$$

We rewrite the probability $\mathbb{P}\left(\exists j \leq n:\left(Z_{i}\right)_{i \leq n} \geq\left(C_{i}\right)_{i \leq n}-\overrightarrow{e_{j}} \cdot 1_{\left\{C_{j}>0\right\}}\right)$ by applying an inclusion-exclusion argument. Corresponding to any $j \leq n$ with $C_{j}>0, Z_{j} \geq C_{j}-1$, and $Z_{i} \geq C_{i}$ for $i \neq j$, we add the term $\mathbb{P}\left(Z_{j} \geq C_{j}-1\right.$, and $Z_{i} \geq C_{i}$ for all $\left.i \neq j\right)$. As a result, we have added those elements with $Z_{i} \geq C_{i}$ for all $i$ a total of $\sum_{i=1}^{n} 1_{\left\{C_{i}>0\right\}}$ many times. Therefore, we compensate by subtracting the term $\left(\sum_{i=1}^{n} 1_{\left\{C_{i}>0\right\}}-1\right) \mathbb{P}\left(\left(Z_{i}\right)_{i \leq n} \geq\right.$ $\left.\left(C_{i}\right)_{i \leq n}\right)$. Further, applying independence of the process $\left(Z_{i}\right)_{i \leq n}$, we have

$$
\begin{aligned}
\mathbb{P}\left(Z_{j} \geq C_{j}-1 \text { and } Z_{i} \geq C_{i} \text { for all } i \neq j\right) & =\mathbb{P}\left(Z_{j} \geq C_{j}-1\right) \mathbb{P}\left(Z_{i} \geq C_{i} \text { for all } i \neq j\right) \\
& =\mathbb{P}\left(Z_{j} \geq C_{j}-1\right) \prod_{\substack{i \neq j, i \leq n}} \mathbb{P}\left(Z_{i} \geq C_{i}\right)
\end{aligned}
$$

\footnotetext{
${ }^{6}$ When Theorem 3.3 is applied in $\S 4$, additional indicator functions will be included to remind us that $\mathbb{P}\left(Z_{i}(n, x) \leq k\right)=0$ if $k<0$.
} 
and $\mathbb{P}\left(\left(Z_{i}\right)_{i \leq n} \geq\left(C_{i}\right)_{i \leq n}\right)=\prod_{i \leq n} \mathbb{P}\left(Z_{i} \geq C_{i}\right)$. Thus, the right hand side of equation (3.2) becomes

$$
1-\sum_{j \leq n}\left(1_{\left\{C_{j}>0\right\}} \mathbb{P}\left(Z_{j} \geq C_{j}-1\right) \prod_{\substack{i \neq j, i \leq n}} \mathbb{P}\left(Z_{i} \geq C_{i}\right)\right)+\left(\sum_{i \leq n} 1_{\left\{C_{i}>0\right\}}-1\right) \prod_{i \leq n} \mathbb{P}\left(Z_{i} \geq C_{i}\right) .
$$

Using the fact that $\mathbb{P}\left(Z_{i} \geq a\right)=1-\mathbb{P}\left(\left(Z_{i} \leq a-1\right)\right)$, expression (3.3) becomes

$$
\begin{aligned}
& 1-\sum_{j \leq n}\left(1_{\left\{C_{j}>0\right\}}\left(1-\mathbb{P}\left(Z_{j} \leq C_{j}-2\right)\right) \prod_{\substack{i \neq j, i \leq n}}\left(1-\mathbb{P}\left(Z_{i} \leq C_{i}-1\right)\right)\right) \\
& +\left(\sum_{i \leq n} 1_{\left\{C_{i}>0\right\}}-1\right) \prod_{i \leq n}\left(1-\mathbb{P}\left(Z_{i} \leq C_{i}-1\right)\right) .
\end{aligned}
$$

The following result shows that only columns with label $\left(C_{i}(n)\right)_{i \leq n}=\overrightarrow{e_{n}}$ have zero pivot mass. In this paper, we will only apply the $(\Leftarrow)$ part of the statement. ${ }^{7}$

Theorem 3.4. For any nonempty collection $L(n)$ of column labels, $\mathcal{P} \mathcal{M}(L(n))=0$ if and only if a column with label $\left(C_{i}(n)\right)_{i \leq n}=\overrightarrow{e_{n}}$ belongs to $L(n)$.

Proof. $(\Leftarrow)$ Given any row label $\left(Z_{i}(n, x)\right)_{i \leq n}$, the vector $\left(C_{i}(n)\right)_{i \leq n}=\overrightarrow{e_{n}}$ satisfies

$$
\begin{aligned}
\sum_{i \leq n}\left(C_{i}(n)-Z_{i}(n, x)\right)^{+} & =\left(C_{n}(n)-Z_{n}(n, x)\right)^{+} \\
& =\left(1-Z_{n}(n, x)\right)^{+} \\
\leq & 1 .
\end{aligned}
$$

Thus, $\mathcal{P} \mathcal{M}\left(\overrightarrow{e_{n}}\right)=0$. Therefore, given $\overrightarrow{e_{n}} \in L(n)$, we have

$$
\mathcal{P} \mathcal{M}(L(n)) \leq \mathcal{P} \mathcal{M}\left(\overrightarrow{e_{n}}\right)=0 .
$$

$(\Rightarrow)$ Now suppose $\overrightarrow{e_{n}} \notin L(n)$. Recall that any column label $\left(C_{i}(n)\right)_{i \leq n}$ satisfies $\sum_{i \leq n} i C_{i}(n)=n$. Since $\overrightarrow{e_{n}}$ is the only column label with $\sum_{i \leq n} C_{i}(n)=1$, this gives us one of two cases for each column label in $L(n)$. Either (a) there exists some $j$ with $C_{j}(n) \geq 2$ or (b) there exists distinct $j, k$ with $C_{j}(n) \geq 1, C_{k}(n) \geq 1$. In case (a), using any row label $\left(Z_{i}(n, x)\right)_{i \leq n}$ with $Z_{j}(n, x)=0$, we have

$$
\sum_{i \leq n}\left(C_{i}(n)-Z_{i}(n, x)\right)^{+} \geq C_{j}(n)-Z_{j}(n, x) \geq 2 .
$$

In case (b), we can take any $\left(Z_{i}(n, x)\right)_{i<n}$ with $Z_{j}(n, x)=Z_{k}(n, x)=0$ to ensure that

$$
\sum_{i \leq n}\left(C_{i}(n)-Z_{i}(n, x)\right)^{+} \geq\left(C_{j}(n)-Z_{j}(n, x)\right)+\left(C_{k}(n)-Z_{k}(n, x)\right) \geq 2 .
$$

Since we have just showed that each column label other than $\vec{e}_{n}$ has a pivot, we use the fact that each of these columns has a pivot in the first row (labeled $\left(Z_{i}(n, x)\right)_{i<n}=$ $(0,0, \ldots, 0))$. Note that $\mathbb{P}(M(n, x)=i)>0$ for all distributions in this paper (see $\S 4)$, so we have

$$
\mathcal{P} \mathcal{M}(L(n)) \geq \mathbb{P}\left(Z_{i}(n, x)=0, \forall i \leq n\right)=\mathbb{P}(M(n, x)=1)>0 .
$$

\footnotetext{
${ }^{7}$ Note that $(\Rightarrow)$ implies that each column label other than $\overrightarrow{e_{n}}$ has pivots. Using equations (2.2)-(2.4) in $\S 2.2$ of [2] (which give the number of columns with label $\overrightarrow{e_{n}}$ in each of these combinatorial settings), we can always determine the number of columns that contain pivots.
} 
The component counting process of a uniform random variable

\section{Pivot mass can be made arbitrarily small for assemblies, multi- sets, and selections}

The following condition on $\mathcal{P} \mathcal{M}$ will be verified for our three combinatorial structures:

$$
\forall n \in \mathbb{N} \forall \varepsilon>0 \exists x(n): x>x(n) \Longrightarrow \text { equation (1.2) holds and } \mathcal{P} \mathcal{M}_{(n, x)}(\cdot)<\varepsilon
$$

\subsection{Assemblies}

In the assembly setting, we can take $Z_{i}(n, x) \sim$ Po $\left(\frac{m_{i} x^{i}}{i !}\right)$ for any $x>0$ to obtain equation (1.2) ( $\$ 2.3$ of [2]). Recall that the CDF of a random variable $Z \sim$ Po $(\lambda)$ is given by $\mathbb{P}(Z \leq k)=\frac{\Gamma(\lfloor k+1\rfloor, \lambda)}{\lfloor k\rfloor !}$ for $k \in \mathbb{Z}_{\geq 0}$, where $\Gamma(a, b)$ is the upper incomplete gamma function - i.e., $\Gamma(a, b)=\int_{b}^{\infty} t^{a-1} e^{-t} d t$.

Lemma 4.1. For a fixed $a>0$, we have $\lim _{b \rightarrow \infty} \Gamma(a, b)=0$.

Proof. Since $\Gamma(a, 0)=\Gamma(a)$ is convergent for $a>0$, we have

$$
\begin{aligned}
\Gamma(a, b) & =\Gamma(a)-\int_{0}^{b} t^{a-1} e^{-t} d t \\
& \rightarrow \Gamma(a)-\Gamma(a) \text { as } b \rightarrow \infty \\
& =0 .
\end{aligned}
$$

We can apply Lemma 4.1 and take $x \rightarrow \infty$ to obtain

$$
\begin{gathered}
\frac{\Gamma\left(C_{i}, \frac{m_{i} x^{i}}{i !}\right)}{\left(C_{i}-1\right) !} \rightarrow 0 \text { when } C_{i}>0, \\
\frac{\Gamma\left(C_{j}-1, \frac{m_{j} x^{j}}{j !}\right)}{\left(C_{j}-2\right) !} \rightarrow 0 \text { when } C_{j}>1 .
\end{gathered}
$$

Therefore, Theorem 3.3 implies that $\mathcal{P} \mathcal{M}(N(n))$ equals

$$
\begin{aligned}
& 1-\sum_{j \leq n}\left(1_{\left\{C_{j}>0\right\}}\left(1-1_{\left\{C_{j}>1\right\}} \frac{\Gamma\left(C_{j}-1, \frac{m_{j} x^{j}}{j !}\right)}{\left(C_{j}-2\right) !}\right) \prod_{\substack{i \neq j, i \leq n}}\left(1-1_{\left\{C_{i}>0\right\}} \frac{\Gamma\left(C_{i}, \frac{m_{i} x^{i}}{i !}\right)}{\left(C_{i}-1\right) !}\right)\right) \\
& +\left(\sum_{i \leq n} 1_{\left\{C_{i}>0\right\}}-1\right) \prod_{i \leq n}\left(1-1_{\left\{C_{i}>0\right\}} \frac{\Gamma\left(C_{i}, \frac{m_{i} x^{i}}{i !}\right)}{\left(C_{i}-1\right) !}\right) .
\end{aligned}
$$

If we let $x \rightarrow \infty$, we can apply (4.2) and (4.3) to deduce that

$$
\mathcal{P} \mathcal{M}(N(n)) \rightarrow 1-\sum_{j \leq n}\left(1_{\left\{C_{j}>0\right\}}(1-0) \prod_{\substack{i \neq j, i \leq n}}(1-0)\right)
$$

\footnotetext{
${ }^{8}$ Although the distribution of $Z_{i}(n, x)$ does not depend on $n$, the choice the process $\left(Z_{i}(n, x)\right)_{i \leq n}$ satisfying (1.2) does depend on $n$. I.e., if $\left(Z_{i}(n, x)\right)_{i \leq n}$ and $\left(Z_{i}(n+1, x)\right)_{i \leq n+1}$ equal $\left(C_{i}(n)\right)_{i \leq n}$ and $\left(C_{i}(n+1)\right)_{i \leq n+1}$, respectively, conditional on the events $\left\{\sum_{i \leq n} i Z_{i}=n\right\}$ and $\left\{\sum_{i \leq n+1} i Z_{i}=n+1\right\}$, respectively, then we need not have $\left(Z_{i}(n, x)\right)_{i \leq n}=\left(Z_{i}(n+1, x)\right)_{i \leq n}$.
} 
The component counting process of a uniform random variable

$$
\begin{aligned}
& +\left(\sum_{i \leq n} 1_{\left\{C_{i}>0\right\}}-1\right) \prod_{i \leq n}(1-0) \\
= & 1-\sum_{j \leq n} 1_{\left\{C_{j}>0\right\}}+\left(\sum_{i \leq n} 1_{\left\{C_{i}>0\right\}}-1\right) \\
= & 0 .
\end{aligned}
$$

This verifies condition (4.1) for assemblies.

\subsection{Multisets}

In the multiset setting, we can take $Z_{i}(n, x) \sim \mathrm{NB}\left(m_{i}, x^{i}\right)$, for any $x \in(0,1)$, to obtain equation (1.2) ( $\{2.3$ of [2]). Recall that the CDF of $Z \sim \mathrm{NB}(r, p)$ is given by $\mathbb{P}(Z \leq k)=1-I_{p}(k+1, r)$, where $I_{p}$ is the regularized incomplete beta function. That is, $I_{x}(a, b)=\frac{B(x ; a, b)}{B(a, b)}$, where $B(a, b)=\int_{0}^{1} t^{a-1}(1-t)^{b-1} d t$, defined for $\operatorname{Re}(a)>0$ and $\operatorname{Re}(b)>0$, is the beta function and $B(x ; a, b)=\int_{0}^{x} t^{a-1}(1-t)^{b-1} d t$ is the incomplete beta function.

Lemma 4.2. Given $a>0, \lim _{x \rightarrow 1} I_{x}(a, b)=1$.

Proof. We have

$$
\begin{aligned}
\lim _{x \rightarrow 1} I_{x}(a, b) & =\lim _{x \rightarrow 1} \frac{B(x ; a, b)}{B(a, b)} \\
& =\lim _{x \rightarrow 1} \frac{\int_{0}^{x} t^{a-1}(1-t)^{b-1} d t}{\int_{0}^{1} t^{a-1}(1-t)^{b-1} d t} \\
& =\frac{\int_{0}^{1} t^{a-1}(1-t)^{b-1} d t}{\int_{0}^{1} t^{a-1}(1-t)^{b-1} d t} \\
& =1 .
\end{aligned}
$$

Using Theorem 3.3, $\mathcal{P M}(N(n))$ equals

$$
\begin{aligned}
& 1-\left(\sum_{j \leq n}\left(1_{\left\{C_{j}>0\right\}}\left(1-1_{\left\{C_{j}>1\right\}}\left(1-I_{x^{j}}\left(C_{j}-1, m_{j}\right)\right)\right) \prod_{\substack{i \neq j, i \leq n}}\left(1-1_{\left\{C_{i}>0\right\}}\left(1-I_{x^{i}}\left(C_{i}, m_{i}\right)\right)\right)\right)\right) \\
& +\left(\sum_{i \leq n} 1_{\left\{C_{i}>0\right\}}-1\right) \prod_{i \leq n}\left(1-1_{\left\{C_{i}>0\right\}}\left(1-I_{x^{i}}\left(C_{i}, m_{i}\right)\right)\right) .
\end{aligned}
$$

Taking $x \rightarrow 1$ and applying Lemma 4.2, we have

$$
\begin{aligned}
\mathcal{P M}(N(n)) \rightarrow & 1-\left(\sum_{j \leq n}\left(1_{\left\{C_{j}>0\right\}}\left(1-1_{\left\{C_{j}>1\right\}}(1-1)\right) \prod_{\substack{i \neq j, i \leq n}}\left(1-1_{\left\{C_{i}>0\right\}}(1-1)\right)\right)\right) \\
& +\left(\sum_{i \leq n} 1_{\left\{C_{i}>0\right\}}-1\right) \prod_{i \leq n}\left(1-1_{\left\{C_{i}>0\right\}}(1-1)\right) \\
= & 1-\sum_{j \leq n} 1_{\left\{C_{j}>0\right\}}+\left(\sum_{i \leq n} 1_{\left\{C_{i}>0\right\}}-1\right) \\
= & 0,
\end{aligned}
$$

which verifies condition (4.1) for multisets. 


\subsection{Selections}

In the selection setting, we can take $Z_{i}(n, x) \sim \operatorname{Bin}\left(m_{i}, \frac{x^{i}}{1+x^{i}}\right), 0<x<\infty$, in order to obtain equation (1.2) ( $\$ 2.3$ of [2]). In our case, we are taking $p=\frac{x^{i}}{1+x^{i}}$, so $p \rightarrow 1$ if and only if $x \rightarrow \infty$. Recall that the $\mathrm{CDF}$ of $Z \sim \operatorname{Bin}(n, p)$ is given by $\mathbb{P}(Z \leq k)=I_{1-p}(n-k, 1+k)$. Using Theorem 3.3, we can express $\mathcal{P} \mathcal{M}(N(n))$ as

$$
\begin{aligned}
& 1-\sum_{j \leq n}\left(1_{\left\{C_{j}>0\right\}}\left(1-1_{\left\{C_{j}>1\right\}} I_{1-p}\left(m_{j}-C_{j}+2, C_{j}-1\right)\right) \prod_{\substack{i \neq j, i \leq n}}\left(1-1_{\left\{C_{i}>0\right\}} I_{1-p}\left(m_{i}-C_{i}+1, C_{i}\right)\right)\right) \\
& +\left(\sum_{i \leq n} 1_{\left\{C_{i}>0\right\}}-1\right) \prod_{i \leq n}\left(1-1_{\left\{C_{i}>0\right\}} I_{1-p}\left(m_{i}-C_{i}+1, C_{i}\right)\right) .
\end{aligned}
$$

Lemma 4.3. We have $\lim _{p \rightarrow 1} I_{1-p}(n-k, 1+k)=0$.

Proof.

$$
\begin{aligned}
\lim _{p \rightarrow 1} I_{1-p}(n-k, 1+k) & =\lim _{p \rightarrow 1} \frac{B(1-p ; n-k, 1+k)}{B(n-k, 1+k)} \\
& =\lim _{p \rightarrow 1} \frac{\int_{0}^{1-p} t^{n-k-1}(1-t)^{k}}{\int_{0}^{1} t^{n-k-1}(1-t)^{k}} \\
& =0 .
\end{aligned}
$$

Using Lemma 4.3, we see that

$$
I_{1-p}=I_{1-\frac{x^{i}}{1+x^{i}}} \rightarrow 0
$$

if $x \rightarrow \infty$. Thus, we apply Theorem 3.3 and Lemma 4.3 while taking $x \rightarrow \infty$ to obtain

$$
\mathcal{P} \mathcal{M}(N(n)) \stackrel{(4.4)}{\rightarrow} 1-\sum_{j \leq n} 1_{\left\{C_{j}>0\right\}}+\left(\sum_{i \leq n} 1_{\left\{C_{i}>0\right\}}-1\right)=0
$$

which verifies condition (4.1) for selections.

\section{Using pivot mass to provide couplings}

Given complete separable metric spaces $S$ and $T$, denote by $p_{S}$ the projection of $S \times T$ onto $S$. Let $\omega$ be a nonempty closed subset of $S \times T$ and $\varepsilon \geq 0$. The following result is Theorem 11 of [6].

Theorem 5.1 (Strassen ${ }^{9}$ ). There is a probability measure $\lambda$ in $S \times T$ with marginals $\mu$ and $\nu$ such that $\lambda(\omega) \geq 1-\varepsilon$, if and only if for all closed sets $L \subseteq T$

$$
\nu(L) \leq \mu\left(p_{S}(\omega \cap(S \times L))\right)+\varepsilon .
$$

Proof of Theorem 1.4. Define $S=\left(\mathbb{Z}_{\geq 0}\right)^{n}$ and $T=\left\{\left(a_{i}\right)_{i \leq n} \in\left(\mathbb{Z}_{\geq 0}\right)^{n}: \sum_{i \leq n} i a_{i}=n\right\}$, corresponding to the set of row labels and the set of column labels respectively, and endow both $S$ and $T$ with the metric $d$ on $\mathbb{Z}^{n}$ defined as $d\left(\left(x_{i}\right)_{i \leq n},\left(y_{i}\right)_{i \leq n}\right):=\max _{i \leq n}\left|x_{i}-y_{i}\right|$. Since $S$ is finite and $T$ is countably infinite, both $S$ and $T$ are separable. In both $S$ and $T$ we have

$$
\left(x_{i}\right)_{i \leq n} \neq\left(y_{i}\right)_{i \leq n} \Longrightarrow d\left(\left(x_{i}\right)_{i \leq n},\left(y_{i}\right)_{i \leq n}\right) \geq 1
$$

\footnotetext{
${ }^{9}$ Thanks to Anthony Quas for suggesting the use of Hall's Marriage Theorem. Strassen's Theorem is a variant of the marriage theorem.
} 
since our $n$-tuples are integer-valued. Therefore, every Cauchy sequence in $S$ (or in $T$ ) converges in $S$ (or in $T$ ). Thus, $S$ and $T$ are complete. Our goal is to apply Theorem 5.1 with $\varepsilon=0$ and

$$
\begin{aligned}
\omega & =P^{c} \\
L & =L(n), \\
\mu_{(n, x)}(i) & =\mathbb{P}(M(n, x)=i), i \in S, \\
\nu_{n}(j) & =\mathbb{P}(N(n)=j), j \in T, \\
\lambda & =p
\end{aligned}
$$

where $P=\{(i, j) \in S \times T:(i, j)$ is a pivot $\}, L(n)$ denotes an arbitrary subset of $T$, and $p$ is our desired joint PMF, with marginals corresponding to $M(n, x)$ and $N(n)$, such that $(i, j) \in P$ implies $p(i, j)=0$. Let us endow $\omega$ with the metric $d_{\omega}$ obtained by restricting the metric

$$
\begin{aligned}
& d_{S \times T}\left(\left(\left(s_{i}\right)_{i \leq n},\left(t_{i}\right)_{i \leq n}\right),\left(\left(s_{i}^{\prime}\right)_{i \leq n},\left(t_{i}^{\prime}\right)_{i \leq n}\right)\right) \\
& :=\max \left(d\left(\left(s_{i}\right)_{i \leq n},\left(s_{i}^{\prime}\right)_{i \leq n}\right), d\left(\left(t_{i}\right)_{i \leq n},\left(t_{i}^{\prime}\right)_{i \leq n}\right)\right)
\end{aligned}
$$

on $S \times T$ to $\omega$. To show that $\omega$ is closed, we first show that $S$ and $T$ are closed. The set $T$ is closed since it is finite. Suppose that $\left(\left(s_{i}(k)\right)_{i \leq n}\right)_{k \in \mathbb{N}}$ is a sequence of $n$-tuples $\left(s_{i}(k)\right)_{i \leq n} \in S$ with $\lim _{k \rightarrow \infty}\left(s_{i}(k)\right)_{i \leq n}=l_{1}$ for some $n$-tuple $l_{1} \in \mathbb{Z}^{n}$. To show that $S$ is closed, it suffices to show that $l_{1} \in S$. For all $\varepsilon^{\prime} \in(0,1)$ there exists a constant $K \in \mathbb{N}$ such that if $k>K$ then $d\left(\left(s_{i}(k)\right)_{i \leq n}, l_{1}\right)<\varepsilon^{\prime}$. Since $\varepsilon^{\prime}<1$, (5.2) implies $l_{1}=\left(s_{i}(K+1)\right)_{i \leq n}$, so $l_{1} \in S$. Therefore, $S$ is closed. Now to show that $\omega$ is closed in $S \times T$, suppose that $\left(\left(s_{i}(k)_{i \leq n}\right),\left(t_{i}(k)\right)_{i \leq n}\right)_{k \in \mathbb{N}}$ is a sequence of pairs $\left(\left(s_{i}(k)_{i \leq n}\right),\left(t_{i}(k)\right)_{i \leq n}\right) \in \omega$ of $n$-tuples $s_{i}(k)_{i \leq n} \in S,\left(t_{i}(k)\right)_{i \leq n} \in T$ with $\lim _{k \rightarrow \infty}\left(\left(s_{i}(k)_{i \leq n}\right),\left(t_{i}(k)\right)_{i \leq n}\right)=\left(l_{1}, l_{2}\right)$, for some $n$-tuples $l_{1}, l_{2} \in \mathbb{Z}^{n}$. Since $S$ and $T$ are closed, we have $l_{1} \in S$ and $l_{2} \in T$. For all $\varepsilon^{\prime} \in$ $(0,1)$ there exists a constant $K \in \mathbb{N}$ such that $k>K \Longrightarrow d_{S \times T}\left(\left(s_{i}(k)_{i \leq n}\right),\left(l_{1}, l_{2}\right)\right)<\varepsilon^{\prime}$. Therefore,

$$
k>K \stackrel{(5.3)}{\Longrightarrow} d\left(\left(s_{i}(k)\right)_{i \leq n}, l_{1}\right), d\left(\left(t_{i}(k)\right)_{i \leq n}, l_{2}\right)<\varepsilon^{\prime} .
$$

Since $\varepsilon^{\prime}<1$,

$$
k>K \stackrel{(5.2)}{\Longrightarrow} d\left(\left(s_{i}(k)\right)_{i \leq n}, l_{1}\right)=d\left(\left(t_{i}(k)\right)_{i \leq n}, l_{2}\right)=0 .
$$

Therefore, applying (5.2) twice, we obtain $\left(l_{1}, l_{2}\right)=\left(\left(s_{i}(K+1)\right)_{i \leq n},\left(t_{i}(K+1)\right)_{i \leq n}\right) \in \omega$, so $\omega$ is a closed subset of $S \times T$. Further, $\omega \neq \emptyset$ since given any column label $j$, the pair $(j, j)$ belongs to $\omega$. Note that the set $L(n)$ is a closed subset of the column labels since $L(n)$ is a finite set. Moreover, $v_{n}(L(n))$ is equal to $\mathbb{P}(N(n) \in L(n))=\frac{\# L(n)}{k_{n}}$, and

$$
\begin{aligned}
\mu_{(n, x)}\left(p_{S}(\omega \cap(S \times L))\right) & =\mathbb{P}\left(M \in p_{S}(\omega \cap(S \times L(n)))\right) \\
& =\mathbb{P}\left(M \in p_{S}\left(P^{c} \cap(S \times L(n))\right)\right) \\
& =\mathbb{P}(\exists j \in L(n):(M, j) \notin P) \\
& =1-\mathcal{P M}_{(n, x)}(L(n)) .
\end{aligned}
$$

Therefore, inequality (5.1) is equivalent to $\frac{\# L(n)}{k_{n}} \leq 1-\mathcal{P M}_{(n, x)}(L(n))$. The latest inequality is equivalent to

$$
\mathcal{P M}_{(n, x)}(L(n)) \leq 1-\frac{\# L(n)}{k_{n}} .
$$


By (4.1), the left hand side can be made arbitrarily small, so (5.4) holds when $1-\frac{\# L(n)}{k_{n}}>0$. When $1-\frac{\# L(n)}{k_{n}}=0$, we must have $L(n)=A_{n}$, so that $\mathcal{P} \mathcal{M}_{(n, x)}(L(n))=0$ by Theorem 3.4. Therefore, by the conclusion of Strassen's Theorem, there exists a joint probability measure $p$, with marginals $\mathbb{P}(M(n, x)=\cdot)$ and $\mathbb{P}(N(n)=\cdot)$, such that $p(\omega)=1$. I.e, the probability of having no pivot in this joint distribution is 1 . Hence, the proof of Theorem 1.4 is complete.

\section{References}

[1] Arratia, R. On the amount of dependence in the prime factorization of a uniform random integer. In B. Bollobás, editor, Contemporary Combinatorics, pp 29-91. Bolyai Society Mathematical Studies, Volume 10, 2002.

[2] Arratia, R., Barbour, A.D., and Tavaré, S. Logarithmic Combinatorial Structures: A Probabilistic Approach. EMS Monographs in Mathematics. European Mathematical Society (EMS), 2003.

[3] Arratia, R. and Tavaré, S. Independent process approximations for random combinatorial structures. Advances in mathematics 104 (1), pp 90-154, 1994.

[4] Duchon, P., Flajolet, P., Louchard, G., and Schaeffer, G. Boltzmann samplers for the random generation of combinatorial structures. Combinatorics, Probability and Computing, 13 (4-5): pp 577-625, 2004.

[5] Quas, A. Reference Request for Couplings with Conditions, URL (version: 2017-10-27): https://mathoverflow.net/q/284525.

[6] Strassen, V. The existence of probability spaces with given marginals. Ann. Math. Statist., Volume 36, Number 2, pp 423-439, 1965.

Acknowledgments. Thanks to Prof. Michael Cranston and Prof. Nathan Kaplan for their support in this project. Thanks to Prof. Richard Arratia and the referee for the helpful suggestions. 